\title{
Trans-Catheter Double-Frequency Ultrasound Ablator for The Treatment of Aortic Valve Leaflets Calcification
}

\author{
Enrico Fermi ${ }^{1 *}$, Davide Benettin ${ }^{1}$, Giacomo Bernava ${ }^{2}$, Maurizio Pesce $^{2}$ and Enrico Pasquino ${ }^{1}$ \\ ${ }^{1}$ AorticLab S r l, Italy \\ ${ }^{2}$ Unità di ricerca in ingegneria tissutale cardiovascolare, Centro Cardiologico Monzino, IRCCS, Italy
}

*Corresponding author: Enrico Fermi, AorticLab S r l, Italy

\section{ARTICLE INFO}

Received: 橎 January 25, 2021

Published: 幽 February 03, 2021

Citation: Enrico Fermi, Davide Benettin Giacomo Bernava, Maurizio Pesce, Enrico Pasquino. Trans-Catheter DoubleFrequency Ultrasound Ablator for The Treatment of Aortic Valve Leaflets Calcification. Biomed J Sci \& Tech Res 33(4)-2021. BJSTR. MS.ID.005429.

Keywords: TDD; Ultrasonic Histotripsy; TAVI, Aortic Stenosis

Abbreviations: AS: Aortic Stenosis; TAVI: Transcatheter Aortic Valve Implantation; TDD: Transcatheter Debridement Device; PZT: Piezoelectric Transducers

\begin{abstract}
Degenerative aortic stenosis (AS) is the most common heart valve disease in developed countries. The prevalence of AS increases with age and varies from $0.2 \%$ at ages 50 to 59 years to $1.3 \%$ at 60 to 69 years, $3.9 \%$ at 70 to 79 years, and $9.8 \%$ at 80 to 89 years [1]. Aortic stenosis is commonly treated with transcatheter aortic valve implantation, (TAVI). But the high cost, some comorbidities, especially in very old age, make the technique impracticable. To overcome these limitations, a Transcatheter Debridement Device (TDD) has been proposed to reduce and remove calcium deposits from the aortic leaflets to restore the valve functionality, for those patients who are not eligible for a TAVI implant or with an initial stenosis in which a valve replacement procedure could be delayed. The TDD technology is based on the use of ultrasound impulsive waves, able to fragment calcium deposits. This technique, called "ultrasonic histotripsy", is implemented through piezoelectric transducers, powered by impulsive waves at two different frequencies. The combination of the two fields allows, in particular, the strengthening of the cavitation forces, a mechanism responsible for the breaking and reduction of calcium deposits. The use of piezoelectric transducers to create low energy waves can be very effective without damaging vital tissues. Through femoral access, the TDD reaches the aortic valve region and there, it is positioned to perform the decalcification treatment.

Similar to lithotripsy, ultrasounds, properly modulated in intensity, frequency, and waveform, can be used to produce fractures and structural changes in calcified deposits. The TDD can also provide an artificial valve function during the debridement treatment of the native valve leaflets, kept open during operation. Numerous tests made on explanted calcific leaflets, have demonstrated the efficacy of the device. Tomographic scans showed the fragmentation and reduction of the deposited calcium, while histological analyses confirmed the integrity and structural coherence of the aortic valve tissue, after the treatment with TDD. Our data suggest that the use of TDD may restore the valve leaflets function, possibly improving the flow with mitigation of ventricular overload and transvalvular pressure gradient. This device could be initially used in non-operable patients and then adopted in a wider proportion of patients with aortic valve stenosis to delay the valve replacement intervention or to prepare the TAVI implantation site.
\end{abstract}

\section{Introduction}

Calcifications of the aortic valve leaflets, due to inflammatory conditions and specific biological processes [1], are quite common after 60 years of age. In particular, aortic valve degenerative stenosis, typical of elderly, has a prevalence of $3 \%$ in the Mediterranean area alone (4.6\% for age $>74$ years) and reaches 10\% in America and North America [1]. The prevalence of a clinically significant stenotic valve condition is about $20 \%$ in patients in the $65-75$ age group, about $35 \%$ in the $75-85$ age group and $48 \%$ in the 85 -age group [2]. The aortic valve is constantly subjected to cyclical mechanical stresses, due to the systo-diastolic motion, with consequent leaflets' stress and to the transvalvular pressure gradient during diastole for each cardiac cycle (about $80 \mathrm{mmHg}$ ). Valve calcification is a gradual accumulation of calcium and phosphate minerals: deposits that can thicken the leaflets creating mineralized structures 
[3]. Dystrophic calcification is concentrated in the areas most subjected to mechanical stress, causing a progressive deterioration of cardiac hemodynamics. The pathogenesis of dystrophic valve calcifications is the result of a chronic inflammatory process and pro-atherosclerotic conditions [3]. To prevent irreversible damage to the heart muscle, calcified aortic valves are replaced by artificial valves, mechanical or bioprosthetic, that can be implanted by conventional open-heart surgery or minimally invasive procedure $[2,4]$.

However, in some cases, surgery may be particularly risky, due to the patient's particular condition (for example, advanced age and other co-morbidities). In case of very old patients with heavy comorbidities, also TAVI is not recommended and the only remaining option is to administer a medical therapy with a low probability of survival at 2 years. It is estimated that more than 200,000 patients, every year, are not treated [4] due to these shortcomings. Patients who are not candidates for surgical or transcatheter prostheses implantation could benefit from a "calcium debridement procedure" of their diseased aortic valve in order to restore, at least in part, the valve functionality. Ultrasonic waves, in particular shockwaves, are daily used to treat calcifications in urological [5,6], or orthopedic fields, and this technique has begun being used for vascular lithotripsy, e.g., at the level of the coronary arteries (10.1016/j.jacc.2020.09.603; 10.2217/fca-2020-0034). To efficiently treat the aortic valve, shockwave generators, external to the body, cannot be sufficiently selective. In fact, in order to achieve an effective lithotripsy, it is necessary to act directly in the site of calcium deposits, without increasing the local temperature that would damage the tissues. It is therefore necessary to use low power energies, while maintaining the calcium breaking capacity. The spaces that can be used to reach the area to be treated are also very limited: for example, if the cardiac valve is to be treated, only arterial catheters, with a diameter of a few millimeters, can be used.

In the present report, we describe the first transcatheter debridement device (TDD) that has been specifically designed to deliver shockwaves to the aortic valve tissue and fracture the accumulated calcium deposits with ultrasound fields generated by opposed piezoelectric transducers at different frequencies [7-9]. The TDD is an ablation device based on physical energies, in particular ultrasound fields that act directly on the calcium deposits present outside and inside the valve leaflets, with the aim at increasing their pliability and restoring sufficient transvalvular flow. Other than for restoring, at least in part, the function of the valve, the TDD device could be also employed to remove calcium deposits from the aortic ring in preparation of conventional or minimally invasive prosthetic valve implantation.

\section{Methods and Materials}

\section{Biophysical Analysis of Ultrasonic Fields Combination}

The technique implemented in the TDD device is the ultrasound histotripsy. Ultrasounds suitably modulated in intensity, frequency, and waveform, can be used to produce fractures and structural changes in calcified deposits. Ultrasonic shockwaves can be generated with piezoelectric generators. The acoustic pressure "bumps" on the calcification, with a force of mechanical stress and, indirectly, by the collapse of cavitation bubbles formed inside the biological tissue [10]. The shockwave is a short duration (20-50 ns) positive pressure pulse, followed by a negative pressure pulse. As described in the literature, these impulse waves, consisting of a positive peak followed by a negative peak, act on calcium deposits with combined effects of forces called spallation at the interface of tissue concretion, shear stress and superfocusing. A particular disruptive action on the deposited calcium, created by ultrasound, is given by the cavitation bubbles [10-13]. The phenomenon of cavitation can be seen as the breaking of a liquid and the consequent formation of bubbles, containing dissolved gas. The action of the ultrasonic field can create acoustic cavitation, distinct in inertial (transient) and non-inertial (stable). When a bubble is exposed to an ultrasonic field, the acoustic pressure acts as an external force that changes its radius. The bubble behaves like an oscillating system with an elasticity given by the gas contained inside it, and an inertia given by the liquid that surrounds the bubble and which oscillates with the wall of the bubble itself.

The bubble therefore has its own frequency which is inversely proportional to its radius (in equilibrium conditions). The relationship between frequency of acoustic field and radius of the bubble can be simplified like this:

$$
f \approx \frac{3}{R}[8]
$$

When the frequency of the acoustic field approaches the proper frequency of the bubble, resonant phenomena occur: the bubble expands during the negative phase of the pressure wave and collapses very quickly and violently, at the arrival of the positive pressure [14]. During the collapse, the bubble can fragment and break or repeat the expansion and collapse cycles. The implosion of the bubbles causes mechanical erosion, due to the release of concentrated energy. The frequencies normally used to induce cavitation, range from tens of $\mathrm{kHz}$ to few $\mathrm{MHz}$. Higher frequencies induce thermal increase, controllable by reducing the amplitude of the wave [14]. For the design of the TDD, we considered low intensity so that the thermal increase is negligible. Cavitation bubbles are inherently unstable, but repetition of impulses creates a "bubble cloud" confined to the focal volume of the ultrasound field [7-9]. The combination of frequencies has shown greater therapeutic efficacy. The hypothesis at the base of a higher efficacy is that the combination of multiple frequencies of the ultrasonic field directly affects cavitation. By combining two frequencies, one about 30 times greater than the other, results an increase in cavitation effect [7]. In particular, low-frequency stimulation contributes by amplifying the effects of cavitation created by the higher frequency, also extending the cavitation volume [7]. 
The number of bubbles is 5 times higher by combining the two frequencies, compared to a frequency only, at equal power supply, while a larger number of bubbles is obtained at lower power [8]. Further enhancement is achieved by increasing the higher frequency, in the range of $\mathrm{MHz}$ [9]. The combination of two sources at two different frequencies increases both the intensity and the bandwidth, "enhancing" the effect of the ultrasound field. Furthermore, non-linear effects combined with the combination of the two frequencies reduce the threshold for generating cavitation effects. The use of frequencies in the $3-4 \mathrm{MHz}$ range allows to act on small diameter bubbles and to exert the action at a lower distance. The higher the frequency, the quicker the signal attenuates, "discharging" the energy at a short distance.

The cavitation enhancement is due to the contribution of some elements [7]:

a. The non-linear effects that lead to the formation of a larger number of bubbles with different rays;

b. The action of the forces of Bjerknes for the intensification of the fragmentation of the bubbles due to the interaction between the bubbles themselves;

c. Formation of new cavitation nuclei due to the lower frequency, caused to implode by the higher frequency (the use of 3 frequencies in this case would further amplify the action)

d. The combination of frequencies makes the mass transfer between the liquid-gas phases more efficient.

\section{TDD}

The Transcatheter Debridement Device includes a set of small piezoelectric transducers (PZT) able to generate local low intensities ultrasound shockwaves, at $100 \mathrm{KHz}$ and $3 \mathrm{MHz}$ frequencies. The piezoelectric transducers (PI Ceramics - Germany) have a size of 3 $\mathrm{mm} \times 10 \mathrm{~mm} \times 1 \mathrm{~mm}$ (Figure 1). The PZT is electrically connected to a Kapton layer and housed in a steel support, to protect the PZT ceramic, to which it is glued. The Kapton layer is an FPC (flexible printed circuit), which electrically isolates the piezoceramic, but which also includes two electrical connection traces to power supply the PZT with the two signals at $3 \mathrm{MHz}$ and $100 \mathrm{KHz}$, applied in sequence, every 6 seconds. The initial radius of the bubbles is a function of the frequency used, for the same value of pressure, according to the equations of Minaret [15] and the approximation of Leighton [16]. Since the duration of these bubbles is greater than the time necessary to vary the frequency of the ultrasounds generated, it is possible to obtain the effect of cavitation that is the expansion and collapse of the bubbles, at different times. The lower frequency field nucleates larger bubbles while the higher frequency field, subsequently applied, creates the cavitation effect with the consequent growth and implosion of the bubbles obtaining a greater efficacy in the treatment of calcium deposits than the use of a single frequency [17-20]. The PZT, are introduced by a catheter, with an outer diameter of $6 \mathrm{~mm}$, designed for access via a femoral artery, reaching the leaflets of the aortic valve.

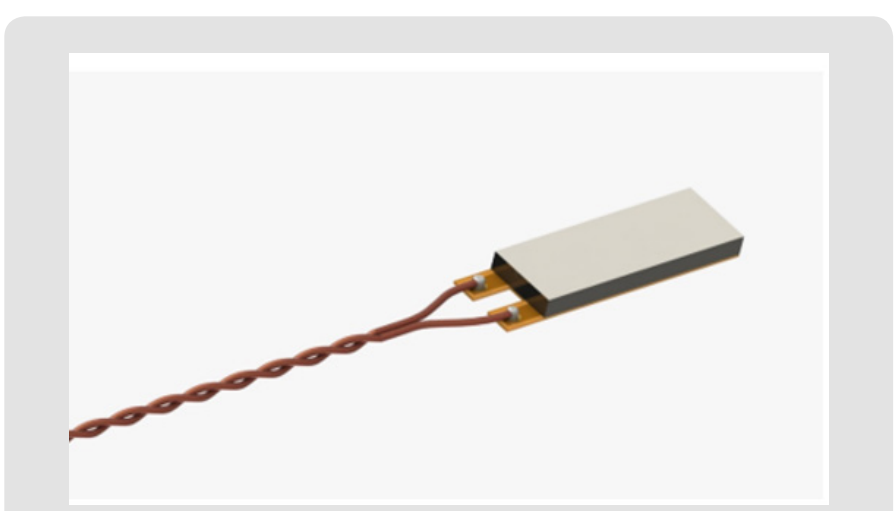

Figure 1: Piezoelectric transducer.

This shell, (Figure 2), is designed to carry electrical connections and provide backing support for wave phase reflection. The whole system is insulated to prevent PZT from generating electrolysis in the environment with consequent emission of free radicals and, in general, to prevent electrocution. A signal generator supplies a pulse wave, (Figure 3), designed to improve the response of the PZT and its capability to pulverize the calcifications. This waveform acts on the transmission environment creating a series of pressure waves: the positive peak creates a compression while the negative one decompresses the environment. The greater speed of the transient implies a greater energy released locally: thanks to this phenomenon, cavitation bubbles are generated which are brought to collapse by the subsequent damping of the waveform. The rapid expansion followed by rapid collapse or implosion of these bubbles, causes fragmentation of the calcified structures. Calcific valve cusps excised during surgical aortic valve replacement were analyzed with micro-CT scan (phoenix v | tome | x m. 3D tomography - country) with $0.19 \mu \mathrm{m}$ pitch, pre- and post-treatment with TDD, to observe the effects on calcium deposits [21-25]. Histological analysis was performed to evaluate the integrity and structural coherence of the healthy aortic valve tissue after ultrasound treatment and to assess the effect on the calcium nodules.

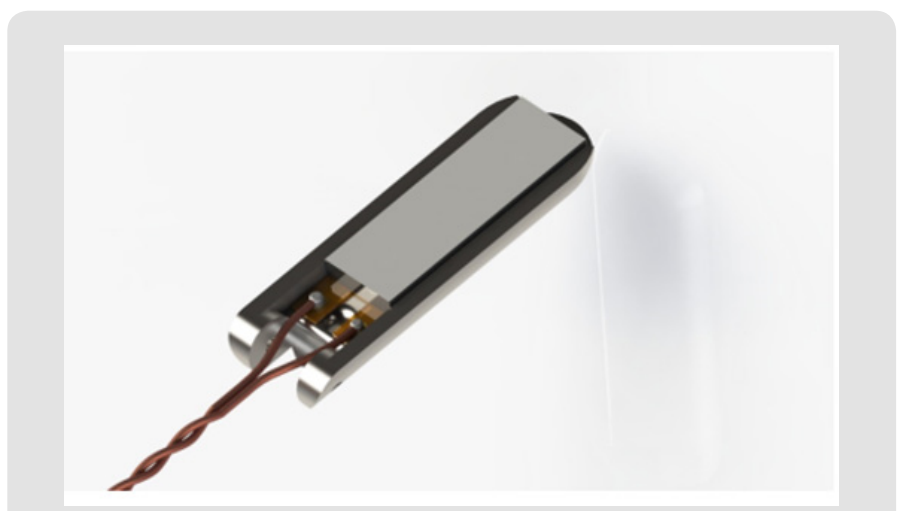

Figure 2: Piezoelectric transducer on the shell. 


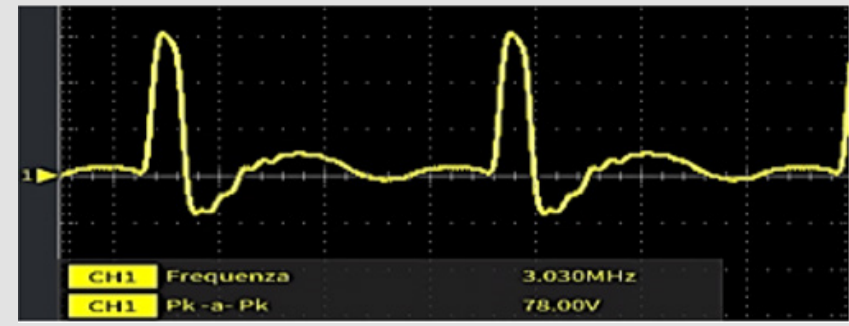

Figure 3: Waveform that supplies the PZT.

The TDD device can also be equipped with an artificial valve function during the debridement treatment of the leaflets of the native valve, kept open during operation (Figure 4).

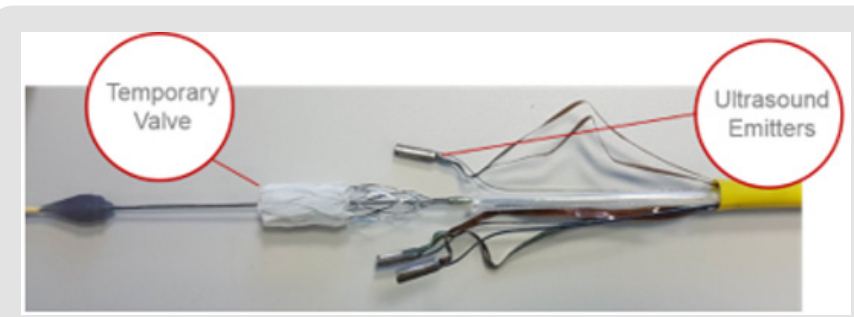

Figure 4: Terminal element of the TDD device.

\section{Results}

Some tests have been performed on explanted human calcific leaflets, to demonstrate the efficacy of the TDD technique, with the purpose to identify the best frequency of the ultrasound field able to maximize the fragmentation and reduction of the calcium deposits. A further goal of the test was to evidence the validity of the biophysical model previously described. Two ultrasound sources at two different frequencies have been combined, placing a couple of piezoceramics transducers on both sides of the aortic valve leaflet. The tests have been performed to show which frequency of the ultrasound field, between $2 \mathrm{MHz}, 3 \mathrm{MHz}$ and $4 \mathrm{MHz}$, combined with the field at a low frequency of $100 \mathrm{kHz}$, produced the greater effects on calcium deposits. For each frequency, a batch of 6 tests have been carried out (18 in all). The CT scans of the leaflets, before and after the treatment, show the percentage of surface area change $(\% \Delta \mathrm{Sm})$ of calcium deposits. The (Figure 5) shows the CT scan of a leaflet, pre (Figure $5 \mathrm{~A}$ ) and post treatment (Figure 5B). The dark grey represents the soft tissue while the light grey / white represents calcium deposits. For tests performed with the $4 \mathrm{MHz}$ frequency we obtain that $\% \Delta \mathrm{Sm}=3.1$ with mean standard error $\sigma^{-}=0.5$. For tests performed with the $3 \mathrm{MHz}$ frequency we obtain that $\% \Delta \mathrm{Sm}=$ 5.3 with mean standard error $\sigma^{-}=0.9$. For tests performed with the $2 \mathrm{MHz}$ frequency we obtain that $\% \Delta \mathrm{Sm}=1.8$ with mean standard error $\sigma^{-}=0.3$. A radar chart (Figure 6) allows to compare the different values of surface areas, showing how the $3 \mathrm{MHz}$ ultrasonic field produces the maximum variations on calcium deposits.

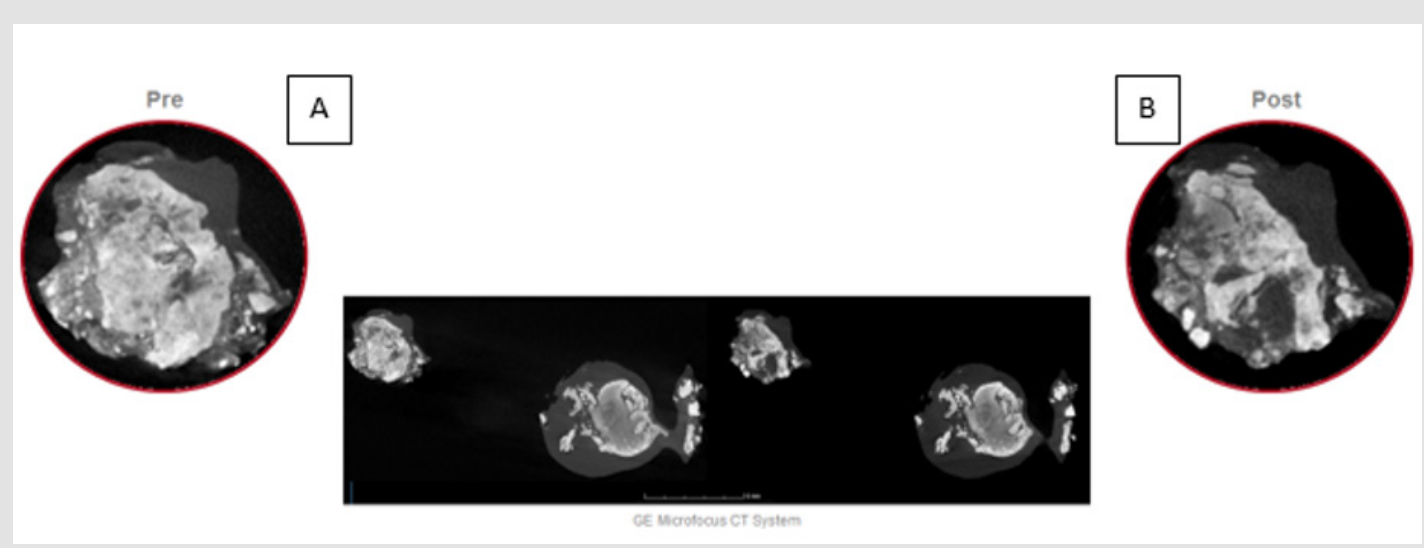

Figure 5: Pre and Post treatment of explanted calcific leaflets.

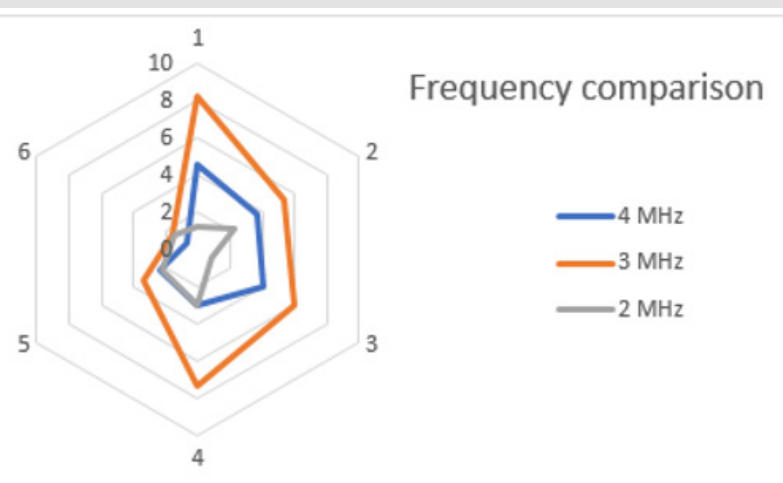

Figure 6: The graph shows the debridement effect on calcium aggregates at different frequencies. 
The preliminary analysis of some histological samples, after TDD treatment, evidenced the effects of the cavitation microbubbles, in the calcium deposits, created by the interaction of the ultrasonic field with the human tissue. The leaflets have been treated with dehydration, inclusion of samples in formalin, sectioning, and histological staining. Von Kossa staining showed residual calcifications spots, with evidence of some "holes" created by the microbubble implosions, (Figure 7), without macroscopic damage to the collagen structures (Figure 8).

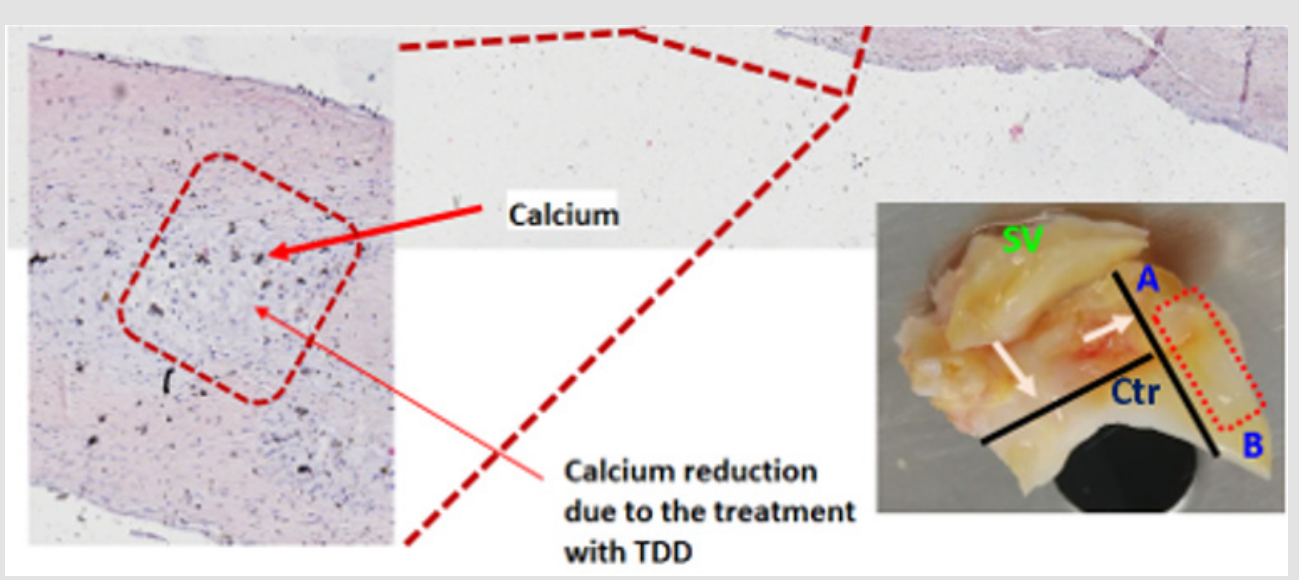

Figure 7: Histological analyzes of the leaflets after the treatment.

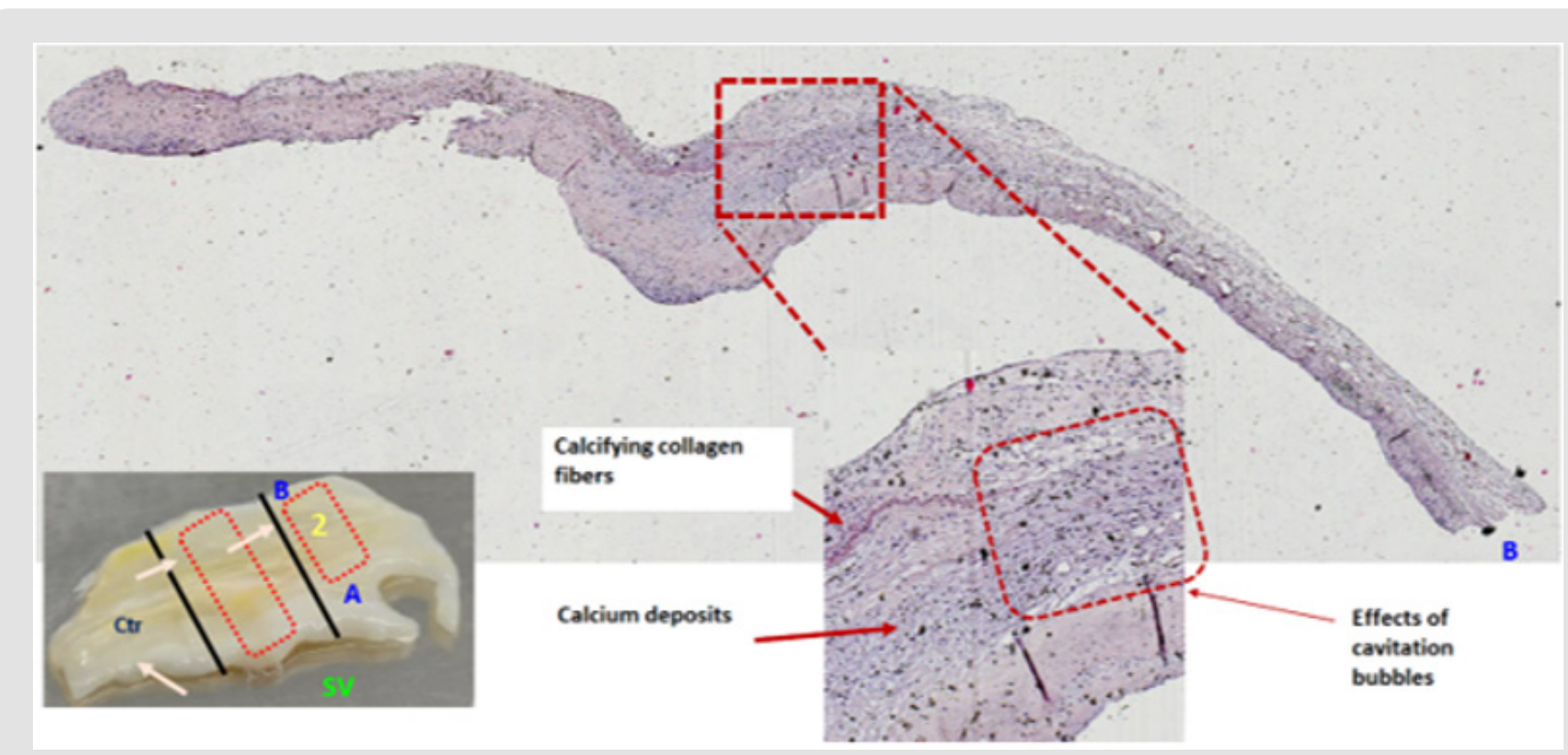

Figure 8: Histological analyzes of the leaflets after the treatment.

\section{Discussion}

Pre and post TDD treatment microtomographic scans demonstrated the fragmentation and reduction of the deposited calcium, while histological analyses confirm the cellular and matrix integrity of the aortic valve leaflets treated with TDD. By reducing and fragmenting the calcium present, the leaflets might recover pliability, restoring, at least partially, the valvular function. In the treated valves there were evidences of micro "holes" in some portions that were calcified before the treatment, and this effect suggests the interaction of the TDD treatment with the biological tissue. The use of a combination of ultrasound frequencies maximized the disruptive effects on calcifications of human aortic valve leaflets.

\section{Conclusion}

The use of a combination of ultrasound frequencies helps to maximizes the disruptive effects on dystrophic calcifications of human aortic valve leaflets. For this reason, TDD seems to be a 
promising technique to reduce the severity of aortic valve stenosis. More tests are needed to better understand the interaction between the ultrasound field and the calcified tissue to demonstrate the clinical efficacy of the treatment.

\section{References}

1. GW Eveborn, H Schirmer, G Heggelund, P Lunde, K Rasmussen (2013) The evolving epidemiology of valvular aortic stenosis. Heart 99(6): 396.

2. BA Carabello, WJ Paulus (2009) Aortic stenosis. Lancet.

3. CI Fisher (2013) Calcific nodule morphogenesis by heart valve interstitial cells is strain dependent Biomechanics and Modeling in Mechanobiology. Biomech Model Mechan 12: 5-7.

4. Otto CM (2017) Aortic stenosis: Treat the patient not the numbers. BMJ Heart 104(3): 190-191.

5. A Nelsius (2017) What is the current status of shock wave lithotripsy? Urologe 56(9): 1147-115.

6. CC Chang, Yong Ren Pu, Ioannis Manousakas, Shen Min Liang, Fan Ming $\mathrm{Yu}$, et al. (2013) In vitro study of the revised ultrasound based real-time tracking of renal stones for shock wave lithotripsy: Part 1. J Urol 189(6): 2357-2363.

7. G Lernetti, P Ciuti, NV Dezhkunov, M Reali, A Francescutto, et al. (1997) Enhancement of high-frequency acoustic cavitation effect by a lowfrequency stimulation. Ultrason Sonochem 4(3): 263-268.

8. R Feng (2002) Enhancement of ultrasonic cavitation yield by multifrequency sonication. Ultrason Sonochem 9(5): 231-236.

9. AL Liu, AM Hsieh (2009) Single-transducer dual-frequency ultrasound generation to enhance acoustic cavitation. Ultrason Sonochem 16(3): 431-438.

10. SW Ohl (2015) Bubbles with shock waves and ultrasound: A review. Interface Focus 5(5): 20150019.

11. M Ashokkumar (2010) The characterization of acoustic cavitation bubbles - An overview. Ultrason Sonochem 18(4): 864-872.

12. M Ashokkumar, Judy Lee, Yasuo Iida, Kyuichi Yasui, Teruyuki Kozuka, et al. (2010) Spatial distribution of acoustic cavitation bubbles at different ultrasound frequencies. Chemphyschem 11(8): 1680-1684.

\section{ISSN: 2574-1241}

DOI: $10.26717 /$ BJSTR.2021.33.005429

Enrico Fermi. Biomed J Sci \& Tech Res

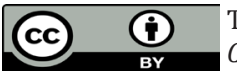

This work is licensed under Creative Commons Attribution 4.0 License

Submission Link: https://biomedres.us/submit-manuscript.php
13. KB Bader (2018) For whom the bubble grows: Physical principles of bubble Nucleation and dynamics in histotripsy ultrasound therapy. Ultrasound in Med \& Biol 45(5): 1056-1080.

14. L Thomas (2016) The fundamentals of power ultrasound- A Review. Acoustics.

15. M Devaud (2008) The Minnaert bubble: An acoustic approach. Eur J Phys 29: 1263.

16. Leighton T (1994) The Acoustic Bubble. Academic Press 96(4): 1994.

17. JM Sinning, E Grube (2017) Unbreak My Heart (Valve) Can Ultrasound Be Used for the Treatment of a Degenerated Surgical Bioprosthesis?. JACC Basic Transl Sci 2(4): 384-385.

18. MJ Matthews, MF Stretanski (2010) Ultrasound Therapy. StatPearls Publishing, Treasure Island, Florida.

19. YA Pishchalnikov, JC Williams, JA Mc Ateer (2011) Bubble proliferation in the cavitation field of a shock wave lithotripter. J Acoust Soc Am 130: E87-E93.

20. X Yang, J Jo (2014) Enhanced cavitation by using two consecutive ultrasound waves at different frequencies. Applied Physics Letters 105(19): 193701.

21. CC Shen (2010) Dual-high-frequency ultrasound excitation on microbubble destruction volume. Ultrasonics 50(7): 698-703.

22. J Luo, Z Niu (2019) Jet and Shock Wave from Collapse of Two Cavitation Bubbles. Scientific Reports 9: 1352.

23. HL Liu, CM Hsieh (2009) Single-transducer dual-frequency ultrasound generation to enhance acoustic cavitation. Ultrasonics Sonochemistry 16: 531-538.

24. P Ciuti, NV Dezhkunov, A Francescutto, AI Kulak, G Iernetti, et al. (2000) Cavitation activity stimulation by low frequency field pulses. Ultrason Sonochem 213-216.

25. José G Díez, (2013) Transcatheter Aortic Valve Implantation (TAVI). Tex Heart Inst J 40(3): 298-301.

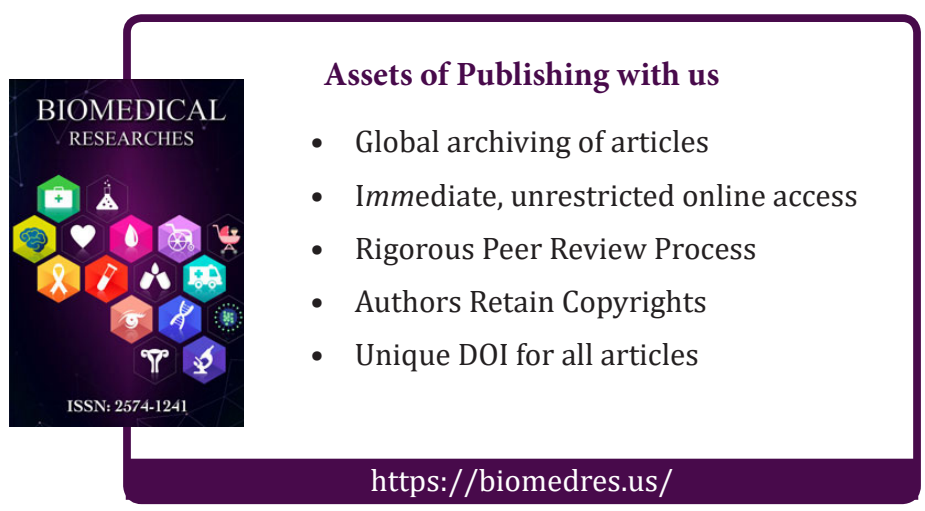

